\title{
OPEN Evaluation of reference genes and characterization of the MYBs in xylem radial change of Chinese fir stem
}

\author{
Kui-Peng Li ${ }^{1}$, Wei Li ${ }^{2}$, Gui-Yun Tao ${ }^{3}$ \& Kai-Yong Huang ${ }^{1 \bowtie}$
}

The radial change (RC) of tree stem is the process of heartwood formation involved in complex molecular mechanism. Chinese fir (Cunninghamia lanceolata (Lamb.) Hook.), an evergreen species, is an important fast-growing timber tree in southern China. In this study, the top four stable genes (IDH, UBC2, RCA and H2B) were selected in RC tissues of 15 years old Chinese fir stem (RC15) and the genes (H2B, 18S, TIP41 and GAPDH) were selected in RC tissues of 30 years old Chinese fir stem (RC30). The stability of the reference genes is higher in RC30 than in RC15. Sixty-one MYB transcripts were obtained on the PacBio Sequel platform from woody tissues of one 30 years old Chinese fir stem. Based on the number of MYB DNA-binding domain and phylogenetic relationships, the CIMYB transcripts contained 21 transcripts of MYB-related proteins (1R-MYB), 39 transcripts of R2R3-MYB proteins (2R-MYB), one transcript of R1R2R3-MYB protein (3R-MYB) belonged to 18 functionannotated clades and two function-unknown clades. In RC woody tissues of 30 years old Chinese fir stem, CIMYB22 was the transcript with the greatest fold change detected by both RNA-seq and qRT-PCR. Reference genes selected in this study will be helpful for further verification of transcript abundance patterns during the heartwood formation of Chinese fir.

Gymnosperms and angiosperms are categorized into woody plants and herbaceous plants. The stem of woody plants is mainly composed of secondary xylem ${ }^{1}$. Wood formation in tree species is performed with the complex coordination of cell differentiation and secondary cell wall (SCW) thickening, which are two highly ordered processes initiated from the vascular cambium ${ }^{2}$. The structure of gymnosperms wood is relatively simple, consisting of tracheids, rays and parenchyma. On the other hand, angiosperm wood is structurally more complex, composed of vessels, fibers, rays, and parenchyma ${ }^{3}$.

The vascular bundle contains phloem cells, cambium cells, and xylem cells from the outer layer to the inner layer of tree stem ${ }^{4}$. Wood developed from several major steps is mainly composed by secondary xylem cells of trees $^{5}$. Sapwood (SW) is derived from vascular cambium has living cells and is located in the outer layers of wood. Heartwood (HW) formation is the process of sapwood senescence with the death of parenchyma cells and the cease of all physiological activity. The transition zone (TZ) is located in between the SW and $\mathrm{HW}^{6}$. Parenchyma tissue contained ray and axial parenchyma cells in secondary xylem represents the bulk of living cells. Ray parenchyma cells are involved in HW formation. Programmed cell death (PCD) plays an important role in secondary xylem cells and HW formation in woody plants ${ }^{4}$.

Gene expression level analysis plays an essential role in molecular biology ${ }^{7}$. Real-time quantitative polymerase chain reaction (qRT-PCR) is a widely applied technique for precise quantification of gene expression and is widely used in modern biology due to its prominent advantages of high sensitivity, specificity, and repeatability ${ }^{8,9}$. Stable internal reference genes are essential to normalize gene expression for obtaining accurate measurement results in the qRT-PCR assay ${ }^{7}$. Reference genes are usually derived from housekeeping genes, which maintain the basic processes of cell cycle ${ }^{10,11}$. However, the housekeeping genes expression levels vary greatly in species, tissues, and under certain experimental conditions ${ }^{12}$. Therefore, selecting stable reference genes according to

${ }^{1}$ Guangxi Key Laboratory of Superior Timber Trees Resource Cultivation and Key Laboratory of Central South Fast-Growing Timber Cultivation of Forestry Ministry of China, Guangxi Forestry Research Institute, Nanning 530002, People's Republic of China. ${ }^{2}$ College of Landscape Architecture and Forestry, Qingdao Agricultural University, Qingdao 266109, People's Republic of China. ${ }^{3}$ Research Institute of Forestry, Chinese Academy of Forestry, Beijing 100091, People's Republic of China. ${ }^{\bowtie}$ email: huangky73@163.com 


\begin{tabular}{|c|c|c|c|c|c|}
\hline Gene symbol & Gene description & Primer sequence (forward/reverse) & Product (bp) & Amplification efficiency (\%) & $\mathbf{R}^{2}$ \\
\hline GAPDH & Glyceraldehyde-3-phosphate dehydrogenase & $\begin{array}{l}\text { GGTCACTGGTTCTGCCAAAT/TGACAACGAGTG } \\
\text { GGGATACA }\end{array}$ & 101 & 88.1 & 0.999 \\
\hline$H 2 B$ & Histone $\mathrm{H} 2 \mathrm{~B}$ & $\begin{array}{l}\text { TATCGGAATTTCCAGCAAGG/ATACCTGGCCAA } \\
\text { TCTGGATG }\end{array}$ & 97 & 92.0 & 0.999 \\
\hline TIP41 & Transcription and replication & $\begin{array}{l}\text { CAAGCCAGGTCTCTCCAAAG/GAGAGCAGGACA } \\
\text { TGGAGGAG }\end{array}$ & 199 & 94.1 & 0.994 \\
\hline $18 S$ & $18 \mathrm{~S}$ ribosomal protein & $\begin{array}{l}\text { GCTTCTTGCTCTACCGGATG/AATGCAACATCA } \\
\text { AGCATGGA }\end{array}$ & 144 & 90.5 & 0.998 \\
\hline$I D H$ & Isocitrate dehydrogenase & $\begin{array}{l}\text { CTTTTCATGCAGTCCCAGGT/TTGCGCTAGCTG } \\
\text { AAGCTGTA }\end{array}$ & 122 & 87.7 & 0.999 \\
\hline PXMP2 & Peroxisomal membrane protein & $\begin{array}{l}\text { TAGTGCAGGCTTGAGGCTTT/AGTTTCCAGTTT } \\
\text { GCCACCAC }\end{array}$ & 159 & 101.4 & 0.999 \\
\hline$R C A$ & Rubisco activase & $\begin{array}{l}\text { GCTTGCCAATGCTCTCTACC/TTTTATTGGGCT } \\
\text { CCAACCAG }\end{array}$ & 208 & 102.7 & 0.996 \\
\hline$U B C 2$ & Ubiquitin-conjugating enzyme & $\begin{array}{l}\text { TTGTTTTGGCAGTCTGCTTG/GCTCGTTTCTGA } \\
\text { TGGCTTTC }\end{array}$ & 216 & 84.6 & 0.998 \\
\hline RPL2 & Large subunit ribosomal protein & $\begin{array}{l}\text { CCGCTGCTCTTTATCCTCAG/GCATCCGAGAGG } \\
\text { GATTATGA }\end{array}$ & 223 & 100.9 & 0.999 \\
\hline DnaJ & Chaperone protein dnaJ & $\begin{array}{l}\text { TGGACCTGGGGATTATGGTA/CATGCCAACTGA } \\
\text { AGCAAAGA }\end{array}$ & 98 & 95.9 & 0.999 \\
\hline$T U B \alpha$ & Tubulin alpha & $\begin{array}{l}\text { CGGAGACTTTTGTGCAGTGA/TCCTGAATGTCG } \\
\text { TGCTTGAG }\end{array}$ & 118 & 87.4 & 0.994 \\
\hline$T U B \beta$ & Tubulin beta & $\begin{array}{l}\text { TGCAGACGAGGATGCTTATG/GCAATTGCAGAA } \\
\text { GCACAGAA }\end{array}$ & 97 & 106.0 & 0.998 \\
\hline
\end{tabular}

Table 1. Primer characteristics of 12 candidate reference genes for qRT-PCR.

the different sample types and various experimental conditions is the primary step for a reliable gene expression analysis by qRT-PCR assay ${ }^{13}$.

Transcription factors (TFs) are proteins capable of controlling the expression of the target genes and play an important role in regulating of multiple biological processes. According to conserved domains, TFs can be divided into different gene families. The MYB family members contain a conserved MYB binding domain consisting of one to four imperfect tandem repeats (R) of about 52 amino acids at the N-terminus. Depending on the number of the adjacent MYB repeats, MYB genes can be classified into four major classes: MYB-related (1R-MYB), R2R3-MYB (2R-MYB), R1R2R3-MYB (3R-MYB), and 4R-like MYB (4RMYB) ${ }^{14}$. MYB proteins, especially R2R3-MYB proteins, are the key factors regulating plant development, primary and secondary metabolism, responses to biotic and abiotic stresses, lignin biosynthesis, xylem secondary cell wall (SCW) formation, and PCD ${ }^{15,16}$.

The radial change $(\mathrm{RC})$ of tree stem is the process of heartwood formation and the process involved in PCD of living cells including ray and axial parenchyma cells. Up to our knowledge, there were few previous studies related to the selection of suitable references genes for target gene expression in RC of tree species stem. Chinese fir (Cunninghamia lanceolata (Lamb.) Hook) is a fast-growing native, allogamous, long-lived tree species mainly distributed in southern China and has been planted for timber production for over 1000 years ${ }^{17}$. Chinese fir accounts for $20-30 \%$ of the total commercial timber production in China ${ }^{18}$. The tree species is a gymnosperm in the cypress family Taxodiaceae. Taxodiaceae traditionally defined includes 10 genera and 16 species $^{19}$. The somatic chromosome number of Chinese fir is $2 n=2 \mathrm{x}=22$ which is in concurrence with other diploid members of Taxodiaceae ${ }^{20}$. The diploid genome sizes of Chinese fir $(28.34 \mathrm{pg} / 2 \mathrm{C})$ is the largest among Taxodiaceae ${ }^{21}$.

In the present study, expression stability of 12 candidate reference genes were assessed by qRT-PCR in RC of Chinese fir stem. Phylogenetic tree was constructed to predict the biological functions of ClMYB transcripts. The expression profile of 61 CIMYB transcripts in the RC were analyzed by RNA-seq. Furthermore, the expression patterns of $25 C l M Y B$ transcripts were validated by qRT-PCR using the four selected reference genes for normalization. This research provides a new understanding of suitable references genes in RC of tree species and expression pattern and biological functions of ClMYB transcripts.

\section{Results}

Expression stability of candidate reference genes. The primers of 12 candidate reference genes were listed in Table 1 . The specificity of each primer pair was verified by $2.0 \%$ agarose gel electrophoresis (Fig. S1) and melting curves analysis (Fig. S2). The primer pair with only a single qRT-PCR product was selected. Amplification efficiency and $\mathrm{R}^{2}$ were counted by the standard curves (Fig. S3). BestKeeper ranks the candidate reference genes according to the Pearson correlation coefficient (r). The reference gene with high stability has a higher $\mathrm{r}$ value. In the radial change of 15 years old Chinese fir stem (RC15), IDH,RCA, UBC2, and RPL2 ranked as the top four stable reference genes (Table 2). In radial change of 30 years old Chinese fir stem (RC30), the results indicated that the $18 \mathrm{~S}, \mathrm{H} 2 \mathrm{~B}, I D H$, and $G A P D H$ were the top four stable genes (Table 3 ).

In the GeNorm analysis, $H 2 B$ and $U B C 2$ had the lowest $M$ values and thus the highest expression stability, followed by Rpl2 and IDH in RC15 tissues (Fig. 1A). In GeNorm analysis of the RC30 tissues, $H 2 B$ and $18 S$ were 


\begin{tabular}{|l|l|l|l|l|l|l|}
\hline Gene & Geometric mean $(\mathbf{C t})$ & Arithmetic mean $(\mathbf{C t})$ & Minimum $(\mathbf{C t})$ & Maximum $(\mathbf{C t})$ & $\begin{array}{l}\text { Coefficient of Variance } \pm \text { Standard } \\
\text { deviation }(\mathbf{C V} \pm \mathbf{S D})\end{array}$ & Pearson correlation coefficient $(\mathbf{r})$ \\
\hline$I D H$ & 25.47 & 25.59 & 23.25 & 32.02 & $7.87 \pm 2.01$ & 0.990 \\
\hline$R C A$ & 26.28 & 26.52 & 22.81 & 34.19 & $10.98 \pm 2.91$ & 0.985 \\
\hline UBC2 & 22.86 & 23.08 & 20.02 & 31.52 & $10.96 \pm 2.53$ & 0.972 \\
\hline RPL2 & 20.18 & 20.43 & 17.68 & 29.27 & $12.89 \pm 2.63$ & 0.964 \\
\hline
\end{tabular}

Table 2. Expression stability of top four stable reference genes in RC15 evaluated by BestKeeper.

\begin{tabular}{|l|l|l|l|l|l|l|}
\hline Gene & Geometric mean $\left(\mathbf{C}_{\mathbf{t}}\right)$ & Arithmetic mean $\left(\mathbf{C}_{\mathfrak{t}}\right)$ & Minimum $\left(\mathbf{C}_{\mathfrak{t}}\right)$ & Maximum $\left(\mathbf{C}_{\mathbf{t}}\right)$ & $\begin{array}{l}\text { Coefficient of Variance } \pm \text { Standard } \\
\text { deviation }(\mathbf{C V} \pm \text { SD })\end{array}$ & Pearson correlation coefficient $(\mathbf{r})$ \\
\hline $18 S$ & 27.24 & 27.30 & 25.25 & 32.33 & $5.56 \pm 1.52$ & 0.989 \\
\hline$H 2 B$ & 23.28 & 23.36 & 21.23 & 28.19 & $6.74 \pm 1.57$ & 0.987 \\
\hline$I D H$ & 24.75 & 24.78 & 23.31 & 28.27 & $4.12 \pm 1.02$ & 0.985 \\
\hline GAPDH & 24.30 & 24.35 & 22.63 & 28.23 & $5.18 \pm 1.26$ & 0.984 \\
\hline
\end{tabular}

Table 3. Expression stability of top four stable reference genes in RC30 evaluated by BestKeeper.
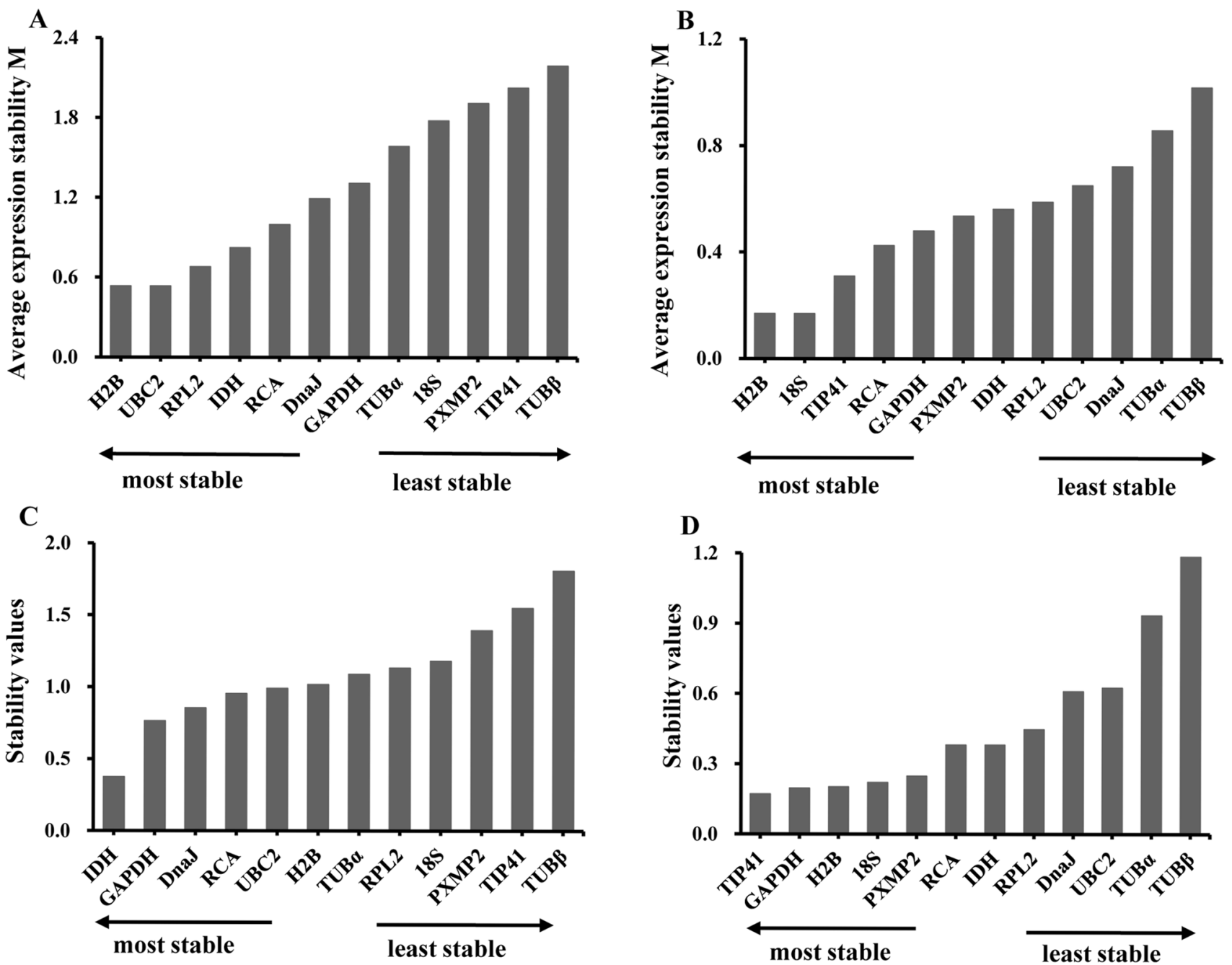

Figure 1. Ranking and expression stability values of the reference genes in RC15 and RC30 tissues by geNorm and NormFinder. (A) Reference genes in RC15 evaluated by geNorm. (B) Reference genes in RC30 evaluated by geNorm. (C) Reference genes in RC15 evaluated by NormFinder. (D) Reference genes in RC30 evaluated by NormFinder. 


\begin{tabular}{|l|l|l|l|l|}
\hline Ranking order & GeNorm & NormFinder & BestKeeper & Comprehensive ranking (mean rank value) \\
\hline 1 & H2B/UBC2 & $I D H$ & $I D H$ & $I D H(1.7)$ \\
\hline 2 & $R P L 2$ & GAPDH & $R C A$ & UBC2 (3.0) \\
\hline 3 & $I D H$ & DnaJ & UBC2 & $R C A(3.3)$ \\
\hline 4 & $R C A$ & $R C A$ & $R P L 2$ & $H 2 B(4.0)$ \\
\hline
\end{tabular}

Table 4. Comprehensive ranking of 12 reference genes in RC15.

\begin{tabular}{|l|l|l|l|l|}
\hline Ranking order & GeNorm & NormFinder & BestKeeper & Comprehensive ranking (mean rank value) \\
\hline 1 & H2B/18S & TIP41 & $18 S$ & H2B (2.0) \\
\hline 2 & TIP41 & GAPDH & H2B & $18 S(2.0)$ \\
\hline 3 & RCA & H2B & IDH & TIP41 (2.7) \\
\hline 4 & GAPDH & $18 S$ & GAPDH & GAPDH $(3.3)$ \\
\hline
\end{tabular}

Table 5. Comprehensive ranking of 12 reference genes in RC30.

the most stable genes, followed by TIP41 and RCA (Fig. 1B). The NormFinder analysis ranked IDH, GAPDH, DnaJ and RCA in the top positions for RC15 samples (Fig. 1C), and in NormFinder analysis of the RC30 tissues, TIP41 exhibited the most stable expression, followed by GAPDH, H2B and $18 S$ (Fig. 1D).

Comprehensive ranking of the reference genes. The ranking order of each reference gene was obtained from 1 (most stable) to 11 (least stable) by geNorm and from 1 (most stable) to 12 (least stable) by BestKeeper and NormFinder. Different ranking of candidate genes among algorithms were revealed in other woody plants $^{22-24}$. According to the arithmetic mean value of the ranking order of each gene, the top 4 stable genes were obtained. $I D H, U B C, R C A$, and $H 2 B$ exhibited higher stability of gene expression in XRC15, and the mean rank value of the genes were 1.7, 3.0, 3.3, and 4.0 respectively (Table 4). $H 2 B(2.0), 18 S(2.0)$, TIP41 (2.7) and GAPDH (3.3) were the top 4 stable genes in XRC30 (Table 5).

Phylogenetic analysis and functional prediction of CIMYB transcripts. To evaluate the evolutionary relationships of the $C l M Y B$ transcripts, an NJ unrooted phylogenetic tree was constructed using amino acid sequences of 61 CIMYBs and 135 AtMYBs (Arabidopsis thaliana) (Fig. 2). Based on the number of MYB DNAbinding domain and phylogenetic relationships, we identified 21MYB-related proteins (1R-MYB), 39 R2R3MYB proteins (2R-MYB), 1R1R2R3-MYB proteins (3R-MYB). According to the alignment result, all MYB members from the two species were subdivided into twenty subgroups (designated as C1-C20). All subgroups were common to the two species. 59 ClMYBs belonging to 18 function-annotated subgroups, and 2 ClMYBs belonging to two function-unknown subgroups were found. Because these ClMYB transcripts were captured from different woody tissues in RC of Chinese fir stem, ClMYB transcripts were involved in divergent functions including stresses response, cell differentiation, lignin biosynthesis, and secondary cell wall formation during heartwood formation of Chinese fir.

R2R3-MYB family genes have been conducted in depth studies and the function of genes are relatively clear $^{25-28}$. To explore the phylogenetic relationships of the R2R3-MYB transcripts, two unrooted phylogenetic trees were constructed with 41 R2R3-MYBs (Chinese fir), 135 R2R3-MYBs (Arabidopsis thaliana), 194 R2R3MYBs (Populus trichocarpa), and 142 R2R3-MYBs (Eucalyptus grandis) by NJ and ML method. A Similar topology was obtained between NJ tree (Fig. S4) and ML tree (Fig. S5). Some homologs between Chinese fir and Arabidopsis thaliana were clustered within the same subgroup may share similar functions. For example, $C l M Y B$ transcripts and AtMYB genes of the C7 subgroup in Fig. 2 (ClMYB18, ClMYB24, ClMYB31, ClMYB41, AtMYB44, and $A t M Y B 73)$ and C8 subgroup (ClMYB13, ClMYB54, ClMYB59, AtMYB33, AtMYB65, and AtMYB101) were clustered within the same subgroup by different methods.

CIMYBs expression profile in RC30 tissues. ClMYB transcript abundance was analysed by RNA-seq in 4 woody tissues of Chinese fir (Fig. 3). Based on RNA-seq expression, 61 ClMYB transcripts revealed 12 expression clusters by $K$-means. Thirty-five $C l M Y B$ transcripts had different expression patterns (fold change $>2$, $P<0.05$ ) in woody tissues (Table S1). Expression of 25 ClMYB transcripts validated by qRT-PCR in the same woody tissues as RNA-seq. Normalized to $H 2 B, 18 S$, TIP41, and GAPDH, a heatmap was constructed with the expression level of ClMYB transcripts (Fig. 4). Based on qPCR-based expression, 25 ClMYB transcripts revealed six expression clusters by K-means.

Three ClMYB transcripts (ClMYB10, ClMYB33, and ClMYB41) had the same expression pattern with the K-means cluster by both qRT-PCR and RNA-seq. Based on six pairwise comparisons in the four woody tissues (X1, X2, X3, and X4), a total of 18 differentially expressed transcripts were identified by RNA-seq (Table S1). Eight differentially expressed transcripts identified by qRT-PCR were also identified by RNA-seq. ClMYB22 was the transcript with the greatest fold change based on RNA-seq data and it was up-regulated with a 6525-fold change 


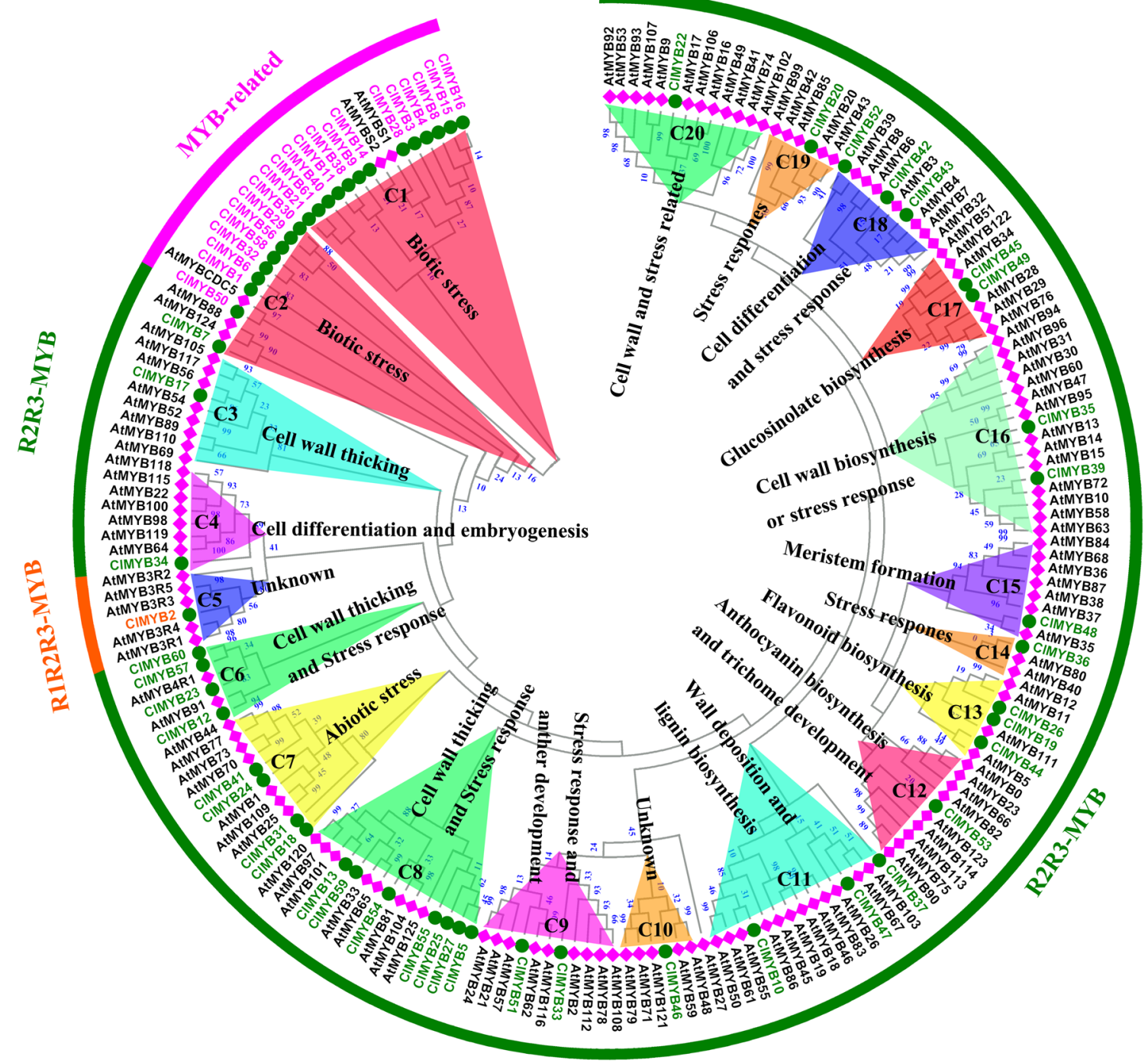

Figure 2. Evolutionary relationships and putative functions of the MYB proteins in Cunninghamia lanceolata (starting as $\mathrm{Cl}$ ) based on the phylogenetic tree with MYB proteins in Arabidopsis thaliana (At). The circular unrooted tree was generated by Neighbor-Joining method in MEGA 7.0 with 1,000 bootstrap replicates, JonesTaylor-Thornton (JTT) model and pairwise deletion treatment. The analysis involved all domain sequences of respective R2R3-MYB protein with 61 ClMYBs and 135 AtMYBs.

in X1 compared to X2. ClMYB22 was also the transcript with the greatest fold change based on qRT-PCR data, but the greatest fold change was up-regulated with an 8714-fold in X1 compared to X4. Nine ClMYB transcripts (ClMYB19, ClMYB21, ClMYB22, ClMYB24, ClMYB26, ClMYB35, ClMYB36, ClMYB42, ClMYB45, ClMYB49, and $C l M Y B 52)$ had same preferential expression detected by two methods. These transcripts all highly expressed in X1, which indicate the results of transcript abundance detected by qRT-PCR were not highly consistent with the RNA-seq in specific xylem tissues (X2, X3, and X4) representing the heartwood formation. Because qRT-PCR and RNA-Seq were conducted with the RNA taken from different increment cores, it reduced the consistency of gene expression patterns detected by the two methods.

\section{Discussion}

In molecular biological research, transcript expression analysis has been an effective basic strategy to understand the establishment of cellular states and predict gene function ${ }^{29}$. qRT-PCR is a commonly used method to quantify gene expression with appropriate reference genes as internal controls and it is unreliable to apply a universal reference gene for different tissues and treatments ${ }^{30}$. HW formation is described as a form of PCD ${ }^{4}$. Expression of genes involved in secondary metabolite biosynthesis and PCD change greatly from SW and TZ toward HW regions $^{24}$. At present, little attention has been paid to selecting stable and appropriate reference genes for the gene expression normalization in RC of gymnosperm.

We determined the expression stability of 12 reference genes in RC of Chinese fir stem by three statistical algorithms. Pearson coefficient of correlation ( $r$ ) was used as the main reference value for gene stability in Bestkeeper. GeNorm recommends selecting genes with an $M$ value $<1.5$. NormFinder provides a stability value of 
A
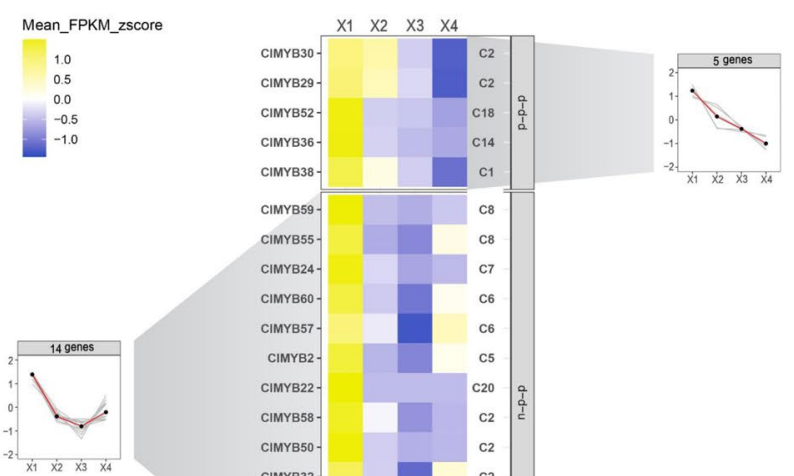

CIMYB59-

CIMYB55.

CIMYB24-

СIMYB60-

CIMYB57.

CIMYB2

CIMYB22-

CIMYB58

CIMYB50-

CIMYB

CIMYB1

CIMYB61-

CIMYB14

CIMYB 34 -

CIMYB5
CIMYB25-

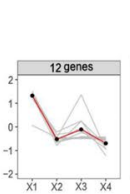

CIMYB31-

CIMYB18.

CIMYB23-

CIMYB42.

CIMYB49-

CIMYB35-

CIMYB26-

CIMYB53-

CIMYB40-

CIMYB51-

CIMYB 33 -

CIMYB54

CIMYB27-

CIMYB17.

CIMYB56-

CIMYB20-

CIMYB45-

Сімүв37-

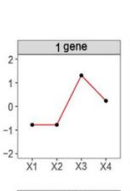

CIMYB28-

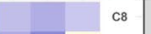

$x_{2} \times 3 \times 4$
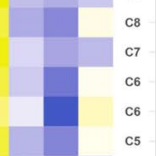

$\mathrm{C} 20$
$\mathrm{C} 2$
$\mathrm{C} 2$
-1
$\mathrm{o}$
$\mathrm{c}$
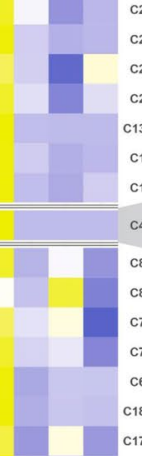

$\mathrm{C} 17$
$\mathrm{C} 16$

$\mathrm{C} 16$
$\mathrm{C} 13$

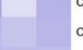

c1
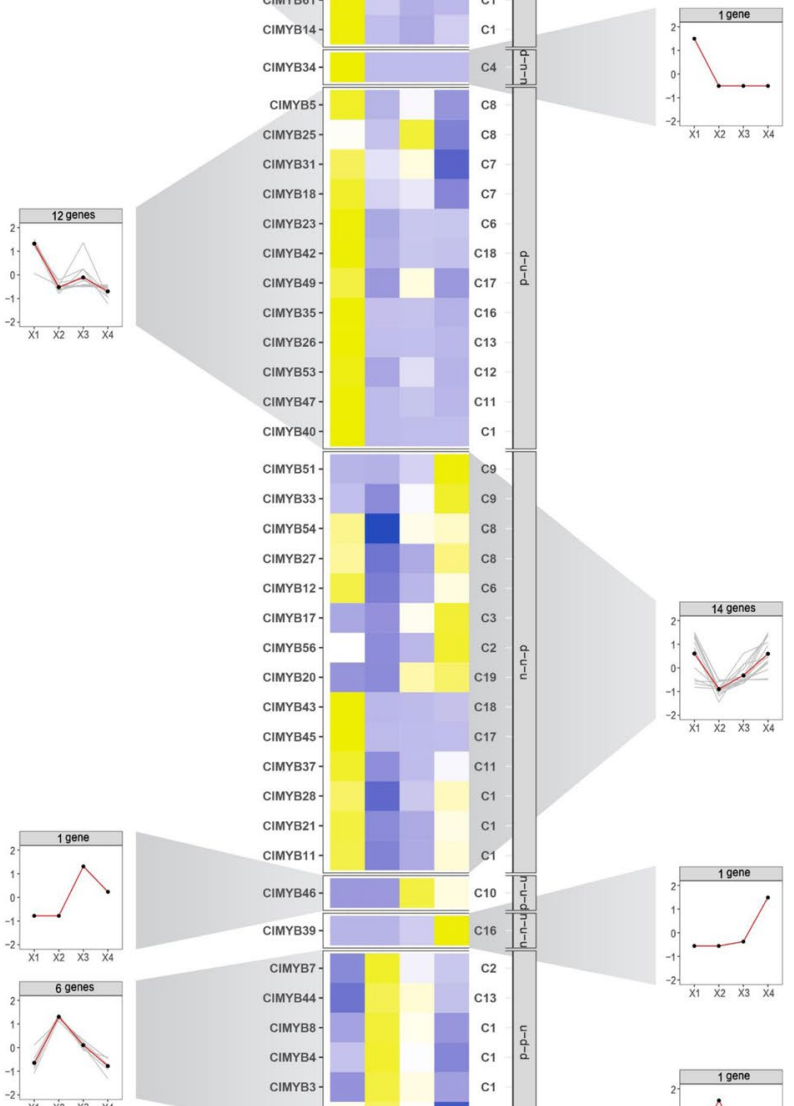

CIMYB39-

CIMYB7-

CIMYB44-

CIMYBB-
CIMYBA-

CIMYB 3 -

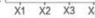

CIMYB15-

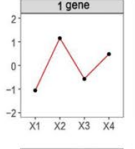

CIMYB16-

CIMYYB13-

CIMYBE.

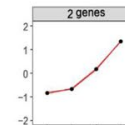

CIMYB48-

CIMYB41-

CIMYB10-

\section{B}

$$
-2 \cdot \dot{x}_{1} \quad \dot{x}_{2} \quad \dot{x}_{3} \quad \dot{x}_{4}
$$

$\mathrm{X} 1 \times 2 \quad \mathrm{X} 3 \quad \mathrm{X} 4$

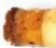

Figure 3. Heatmap of the RNAseq transcript abundance pattern of the $61 \mathrm{MYB}$ transcripts from Cunninghamia lanceolata in four woody tissues clustered in 12 expression groups by K-means. (A) Heatmap of the RNAseq transcript abundance pattern. Transcript name is included to the left of the heatmap and the short name of the phylogenetic subgroup is shown to the right. K-means clusters were performed based on fragments per kilobase of exon per million fragments mapped (FPKM) values adjusted through z-score standardization. (B) Localization of different woody tissues used for RNA-seq and qRT-PCR experiment of 30 years old Chinese fir stem. X1, cambium zone; X2, outer sapwood; X3, inner sapwood; X4, transition zone. 


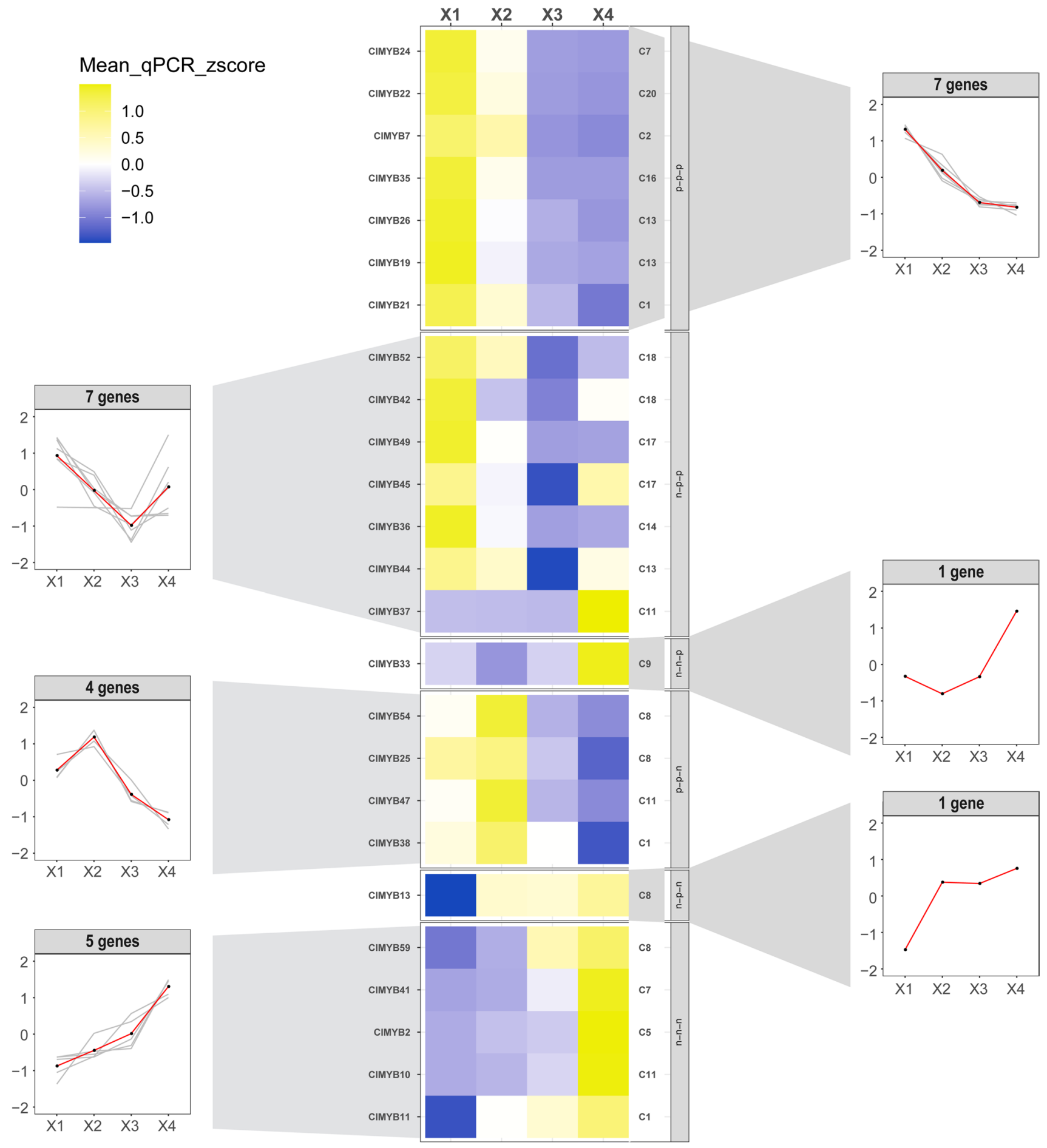

Figure 4. The heatmap of the transcript abundance patterns validated by qRT-PCR of the 25 MYB transcripts from Cunninghamia lanceolata in four woody tissues. Twenty-five ClMYB transcripts were clustered in 6 expression groups by K-means. Data was analyzed by the $2^{-\Delta \Delta \mathrm{Ct}}$ method and adjusted through z-score standardization. Transcript name is included to the left of the heatmap and the short name of the phylogenetic subgroup is shown to the right.

each reference gene by estimating both intra- and inter- group expression variation ${ }^{12,32,33}$. According to the three algorithms, these results show that the expression of the candidate genes is more variable in RC15 than in RC30. The accuracy of target gene expression level may be influenced by using just a single reference gene in qRT-PCR ${ }^{7}$. Integrated ranking of reference genes stability from the three software and utilizing multiple reference genes for the normalization is necessary and shows up as a more reliable accuracy of target gene expression level in 
qRT-PCR ${ }^{34}$. Compared to the calculation of geometric mean, arithmetical mean of ranking values by the different algorithms is a more comprehensive way to create a consensus ranking ${ }^{35}$.

In our study, $I D H, U B C 2, R C A$, and $H 2 B$ were the most stable reference genes in RC15. $H 2 B, 18 S, T I P 41$, and $G A P D H$ were the most stable reference genes in $\mathrm{RC} 30$. $H 2 B$ was considered as the stable reference genes from adventitious rooting in Eucalyptus globulus ${ }^{36}$. IDH was used as reference genes in gene expression experiments to analyze flowers of Pinus massoniana L. in different developmental phases ${ }^{37}$. GAPDH was the suitable reference gene for xylem tissues in Camellia oleifera ${ }^{38} .18 S$ was one of stable reference genes in different tissues of Taxus $s p p^{39}$. UBC2 was identify the best reference genes for Osmanthus fragrans ${ }^{40}$. $R C A$ was upregulated in response to salt stress for cucumber ${ }^{41}$. TIP41 was the stable reference gene for low-temperature stress in Populus trichocarp $p^{42}$.

Vascular plants developed specialized conductive tissues in xylem vessels characterized by SCW deposition to increase water conductive activity ${ }^{43}$. MYBs play critical roles in controlling xylem cell differentiation and SCW biosynthesis pathway ${ }^{44}$. Homology classification is important to predict the gene function and genes in the same subgroup based on the sequence are thought to have relatively similar roles ${ }^{45}$. Three MYB subgroups (S5, S6 and SAtM5) are identified by phylogenetic approach to belonging to putative woody-expanded subgroups from the woody perennial species. These woody-expanded subgroups are involved in the phenylpropanoid pathway ${ }^{27}$. ClMYB26 and ClMYB49 were identify to belong to S5. ClMYB19 and ClMYB36 belong to SAtM5. These four MYB transcripts from Chinese fir were preferentially expressed in the cambium-enriched region of Chinese fir and may be involved in regulating of the phenylpropanoid pathway.

ClMYB transcript and AtMYB genes of the C3 subgroup (ClMYB17, AtMYB56, AtMYB105, and AtMYB117) were clustered into the same subgroup with different methods. These MYB genes of Arabidopsis are involved in the cell wall metabolism ${ }^{46,47}$ and organ patterning ${ }^{48,49}$. ClMYB17 may have similar functions and was preferentially expressed in the transition zone of Chinese fir. Some ClMYB transcripts and AtMYB genes of C8 subgroup (ClMYB13, ClMYB54, ClMYB59, AtMYB33, AtMYB65, and AtMYB101) were clustered into the same subgroup. These MYB genes of Arabidopsis are involved in the programmed cell death process ${ }^{50}$. Although ClMYB13, ClMYB54, and ClMYB59 maybe involved in programmed cell death process, the transcripts with different expression patterns in radial change of Chinese fir stem have different functions.

Some ClMYB transcripts and AtMYB genes of C2 subgroup (ClMYB7, AtMYB88, and AtMYB124), C7 subgroup (ClMYB18, ClMYB24, ClMYB31, ClMYB41, AtMYB44, and AtMYB73), C9 subgroup (ClMYB33, ClMYB51, AtMYB62, and AtMYB108), C16 subgroup (ClMYB39, AtMYB13, AtMYB14, and AtMYB15), C18 subgroup (ClMYB42, ClMYB43, ClMYB52 and AtMYB4), C19 subgroup (ClMYB20 and AtMYB20) were clustered into each subgroup. $A t M Y B$ genes of these subgroups involve in transducing or conferring resistance to abiotic stresses ${ }^{51-56}$. ClMYB transcripts of these subgroups may have similar functions. ClMYB18, ClMYB20, ClMYB24, ClMYB31, ClMYB52, ClMYB42, and ClMYB43 were preferentially expressed in the cambium zone of Chinese fir. ClMYB7 was highly expressed in outer sapwood. ClMYB33, ClMYB39, ClMYB41, and ClMYB51 showed higher expression in the transition zone. The different expression pattern of these ClMYBs indicates the transcripts involved in stress responses at the different stages during heartwood formation of Chinese fir.

\section{Methods}

Collection of plant material. Samples excised at breast height $(1.3 \mathrm{~m})$ of 26 years old Chinese fir stem were successfully used to study cellular changes from cambium to heartwood in Chinese fir $^{57}$. Increment cores were taken from 35 years old Taiwanina (Taiwania cryptomerioides Hayata) stems to analyze the expression profiling of the heartwood formation process ${ }^{31}$. In this study, increment cores were sampled from breast height of three well-grown individuals (of each) of 15 years old and 30 years old Chinese fir stems in summer (August 2019). The radial change tissues of outer sapwood, inner sapwood, and the transition zone were taken from 15 years old Chinese fir stems. The tissues of outer sapwood, middle sapwood, inner sapwood, and the transition zone were taken from 30 years old Chinese fir stems. While these above samples were collected, all samples were immediately frozen in liquid nitrogen and later stored in $-80^{\circ} \mathrm{C}$ for subsequent experiments. Increment cores were taken from our test plantation in Rongshui county of China $\left(25^{\circ} 04^{\prime} 48.6^{\prime \prime} \mathrm{N}, 108^{\circ} 15^{\prime} 16.3^{\prime \prime} \mathrm{E}\right)$. Since the collected materials were taken from our (own) test plantation, there will be no permission needed. The study complies with relevant institutional, national, and international guidelines and legislation.

Total RNA extraction and cDNA synthesis. Total RNA from different samples was isolated by RNAprep Pure Plant Kit (Tiangen, China) without DNA contamination following the manufacturer's protocol. RNA integrity was checked on $1 \%$ agarose gel electrophoresis. The quantity and quality of total RNA were measured by a NanoDrop 2000 Spectrophotometer (Thermo Scientific, US). RNA was homogenized with RNase-free water. Total RNA (500 ng per samples) was reversely transcribed to first-strand of cDNA by PrimeScript RT reagent Kit (TaKaRa, Japan). All cDNA samples were stored at $-20{ }^{\circ} \mathrm{C}$.

qRT-PCR. The full-length transcriptome from equal amounts of mixed RNA obtained from 4 woody tissues (X1, X2, X3, and X4) of one 30 years old Chinese fir was sequenced on the PacBio Sequel platform from our laboratory (SRA accession: PRJNA760952). The consensus sequence was obtained. A total of 12 RNA-seq libraries, with 4 woody tissues (X1, X2, X3, and X4) per tree for three 30 years old individuals were sequenced by Illumina HiSeqX platform (SRA accession: PRJNA760952). According to the literature of reference genes selection $^{36,58,59}$ and our previous RNA-Seq data of 4 woody tissues (Novogene, China), 12 candidate reference genes were screened based on rules of q-value $\geq 0.05, F P K M \geq 5$, and Fold Change $<2$. Specificity of primer pair of 12 candidate reference genes and 25 ClMYB transcripts were controlled by performing melting curves of the qRT-PCR products (Tianlong, China). Melting curves showed a single peak indicates the absence of side products and primer dimers. 
Nucleotide sequence data of 12 candidate reference genes and the primers of 25 ClMYBs were listed in Table S2. The conserved domains of their mRNA sequences were identified using the NCBI database and local blast of PacBio-Seq data, the primers for qRT-PCR amplification were then designed on the web using Primer 3.0 (http://www.primer3plus.com/primer3web/primer3web_input.htm) with the following criteria: the primers should be with limited lengths of 20-24 bp, melting temperatures (Tm) in the range of $55-62^{\circ} \mathrm{C}$, GC contents varying from 45 to $55 \%$, and product lengths of $80-250 \mathrm{bp}$, other parameters were set as default. qRT-PCR assay was performed in a volume of $20 \mu \mathrm{L}$ with three technical replicates on Gentier 32R real-time PCR system (Tianlong, China) using TB Green Premix Ex Taq (TaKaRa, Japan). The reaction was performed at the following conditions: initial denaturation at $95^{\circ} \mathrm{C}$ for $30 \mathrm{~s}$, followed by 45 cycles of $95^{\circ} \mathrm{C}$ for $5 \mathrm{~s}, 55^{\circ} \mathrm{C}$ for $10 \mathrm{~s}$, and $72{ }^{\circ} \mathrm{C}$ for $10 \mathrm{~s}$.

Phylogenetic analysis of the CIMYB proteins. The full-length transcriptome of 4 woody tissues (X1, $\mathrm{X} 2, \mathrm{X} 3$, and X4) from one 30-year-old Chinese fir was obtained on the PacBio Sequel platform (SRA accession: PRJNA760952). To derive annotation information and identify the MYB transcripts, the unique isoforms were mapped to Swiss-Prot (https://www.uni-prot.org/uniprot/). The online software SMART (http://smart.embl.de/) were used to identify the domain sequences of all MYB proteins. The names and accession numbers of MYBs in the other three species were based on previous research ${ }^{25-28}$. According to accession numbers, the protein sequence of MYBs was available in Arabidopsis thaliana (Plant Transcription Factor Database: http://planttfdb. gao-lab.org/), Populus trichocarpa (PoplarGene: http://bioinformatics.caf.ac.cn/PoplarGene), and Eucalyptus grandis (NCBI Genbank: https://www.ncbi.nlm.nih.gov/genbank/). The online SMART software (http://smart. embl.de/) were used to identify the amino acid sequence of domains of each MYB protein. The protein sequence of 61 ClMYBs and the whole domain of each MYB protein are shown in Table S3.

Based on the whole domain sequence of each MYB protein, neighbor-joining (NJ) phylogenetic trees were generated by MEGA 7.0 with 1,000 bootstrap replicates, Jones-Taylor-Thornton (JTT) model and pairwise deletion treatment. Based on the whole domain sequence of each MYB protein, Maximum Likelihood (ML) phylogenetic tree was generated by MEGA 7.0 with 1,000 bootstrap replicates, Jones-Taylor-Thornton (JTT) model and complete deletion treatment. The biological functions of ClMYBs were predicted by homologous MYBs with validated function within the same phylogenetic tree clade.

RNAseq and qRT-PCR expression analysis. The correlation coefficient $\left(\mathrm{R}^{2}\right)$ and amplification efficiency (E) of primer pairs were counted by the linear regression model with a series of fivefold dilution of first-strand cDNA $(0.5 \mu \mathrm{g} / \mu \mathrm{L})$. The PCR efficiency was calculated by the equation $\mathrm{E}=\left(10^{[-1 / \mathrm{slope}]}-1\right) \times 100 \%$. The expression stability of candidate reference genes was calculated using geNorm, NormFinder, and Bestkeeper ${ }^{12,55,56}$. We used geometric mean of 4 selected reference genes of Chinese fir for the normalization of data. The relative expression levels were evaluated by $2^{-\Delta \Delta C T}$.

RNA-seq data from 4 different woody tissues (X1, X2, X3, and X4) per tree for three 30 years old individuals were sequenced by Illumina HiSeqX platform (SRA accession: PRJNA760952). Mean fragments per kilobase of exon per million fragments mapped (FPKM) values per transcript were calculated for each tissue. K-means clusters performed with Perl were based on FPKM values adjusted through z-score standardization. Six pairwise comparisons were performed to identify DEGs: (1) X1 versus X2; (2) X1 versus X3; (3) X1 versus X4; (4) X2 versus X3; (5) X2 versus X4; and (6) X3 versus X4. Differential expression analysis by RNA-seq and qRT-PCR were based on rules of fold change $>2$ and $P<0.05$. Analysis of $z$-score standardization and ANOVA and drawing of heatmap and trendline was performed by $\mathrm{R}$ package.

Received: 6 April 2021; Accepted: 14 December 2021

Published online: 07 January 2022

\section{References}

1. Li, X., Wu, H. X. \& Southerton, S. G. Comparative genomics reveals conservative evolution of the xylem transcriptome in vascular plants. BMC Evol. Biol. 10, 1-14 (2010).

2. Zhao, Y. et al. Intron-mediated alternative splicing of PtrWND1B regulates cell wall thickening during fiber development in Populus. Plant Physiol. 164, 765-776 (2014).

3. Zhang, M. et al. Comparison of wood physical and mechanical traits between major gymnosperm and angiosperm tree species in China. Wood Sci. Technol. 51, 1405-1419 (2017).

4. Spicer, R. Senescence in secondary xylem: heartwood formation as an active developmental program. In Vascular transport in plants (eds Holbrook, N. M. \& Zwieniecki, M. A.) 457-475 (Elsevier Academic Press, 2005).

5. Plomion, C., Leprovost, G. \& Stokes, A. Wood formation in trees. Plant Physiol. 127, 1513-1523 (2001).

6. Kampe, A. \& Magel, E. New insights into heartwood and heartwood formation. In Cellular aspects of wood formation 71-95 (Springer, Berlin/Heidelberg, 2013).

7. Vandesompele, J. et al. Accurate normalization of real-time quantitative RT-PCR data by geometric averaging of multiple internal control genes. Genome Biol. 3, 1-11 (2002).

8. Kulcheski, F. R. et al. The use of microRNAs as reference genes for quantitative polymerase chain reaction in soybean. Anal. Biochem. 406, 185-192 (2010).

9. Li, Q., Fan, C. M., Zhang, X. M. \& Fu, Y. F. Validation of reference genes for real-time quantitative PCR normalization in soybean developmental and germinating seeds. Plant Cell Rep. 31, 1789-1798 (2012).

10. Plachetka-Bożek, A. \& Augustyniak, M. Evaluation of candidate reference genes for quantitative gene expression analysis in Spodoptera exigua afer long-time exposure to cadmium. Sci. Rep. 7, 8338 (2017).

11. Sun, H. P., Li, F., Ruan, Q. M. \& Zhong, X. H. Identification and validation of reference genes for quantitative real-time PCR studies in Hedera helix L. Plant Physiol. Biochem. 108, 286-294 (2016).

12. Radonic, A. et al. Guideline to reference gene selection for quantitative real-time PCR. Biochem. Biophys. Res. Commun. 313, 856-862 (2004). 
13. Saha, P. \& Blumwald, E. Assessing reference genes for accurate transcript normalization using quantitative real-time PCR in pearl millet [Pennisetum glaucum (L.) R. Br]. PLoS ONE 9, e106308 (2014).

14. Liu, C., Hao, J., Qiu, M., Pan, J. \& He, Y. Genome-wide identification and expression analysis of the MYB transcription factor in Japanese plum (Prunus salicina). Genomics 112, 4875-4886 (2020).

15. Hirai, R. et al. The progression of xylem vessel cell differentiation is dependent on the activity level of VND7 in Arabidopsis thaliana. Plants (Basel). 9, 39 (2019)

16. Xie, M. et al. Regulation of lignin biosynthesis and its role in growth-defense tradeoffs. Front. Plant Sci. 28, 1427 (2018).

17. Ma, X. et al. Nutrient cycling and distribution in different-aged plantations of Chinese fir in southern China. Forest Ecol. Manage. 243, 61-74 (2007).

18. Bian, L. et al. Spatial analysis increases efficiency of progeny testing of Chinese fir. J. For. Res. 28, 445-452 (2017).

19. Brunsfeld, S. J. et al. Phylogenetic relationships among the genera of Taxodiaceae and Cupressaceae: evidence from rbcL sequences. Syst. Bot. 19, 253-262 (1994).

20. Khoshoo, T. N. Chromosome numbers in gymnosperms. Silvae Genetica. 10, 1-9 (1961).

21. Hizume, M. et al. Flow cytometric determination of genome size in the Taxodiaceae Cupressaceae sensu stricto and Sciadopityaceae. Cytologia 66, 307-311 (2001).

22. Imai, T. H. et al. Evaluation of reference genes for accurate normalization of gene expression for real time quantitative PCR in Pyrus pyrifolia using different tissue samples and seasonal conditions. PLoS ONE 9, 1-11 (2014).

23. Lin, Y. L. \& Lai, Z. X. Reference gene selection for qPCR analysis during somatic embryogenesis in longan tree. Plant Sci. 178, $359-365$ (2010).

24. Li, H. P. et al. Screening of valid reference genes for real-time RT-PCR data normalization in Hevea brasiliensis and expression validation of a sucrose transporter gene HbSUT3. Plant Sci. 181, 132-139 (2011).

25. Chen, Y. H. et al. The MYB transcription factor superfamily of Arabidopsis: expression analysis and phylogenetic comparison with the rice MYB family. Plant MolBiol. 60, 107-124 (2006).

26. Stracke, R., Werber, M. \& Weisshaar, B. The R2R3-MYB gene family in Arabidopsis thaliana. Curr. Opin. Plant Biol. 4, 447-456 (2001).

27. Soler, M. et al. The Eucalyptus grandis R2R3-MYB transcription factor family: evidence for woody growth-related evolution and function. New Phytol. 206, 1364-1377 (2015).

28. Wilkins, O., Nahal, H., Foong, J., Provart, N. J. \& Campbell, M. M. Expansion and diversification of the Populus R2R3-MYB family of transcription factors. Plant Physiol. 149, 981-993 (2009).

29. Gonzales, K. A. \& Ng, H. H. Biological networks governing the acquisition, maintenance, and dissolution of pluripotency: insights from functional genomics approaches. Cold Spring HarbSymp. Quant. Biol. 80, 189-198 (2015).

30. Dheda, K. et al. The implications of using an inappropriate reference gene for real-time reverse transcription PCR data normalization. Anal. Biochem. 344, 141-153 (2005).

31. Yeh, T. F., Chu, J. H., Liu, L. Y. \& Chen, S. Y. Differential gene profiling of the heartwood formation process in Taiwania cryptomerioides Hayata xylem tissues. Int. J. Mol. Sci. 21, 960 (2020).

32. Pfaffl, M. W., Tichopad, A., Prgomet, C. \& Neuvians, T. P. Determination of stable housekeeping genes, differentially regulated target genes and sample integrity: BestKeeper-Excel-based tool using pair-wise correlations. Biotechnol. Lett. 26, 509-515 (2004).

33. Andersen, C. L., Jensen, J. L. \& Orntoft, T. F. Normalization of real-time quantitative reverse transcription-PCR data: A model-based variance estimation approach to identify genes suited for normalization, applied to bladder and colon cancer data sets. Cancer Res. 64, 5245-5250 (2004).

34. Yao, S. C. et al. Identification and validation of reference genes for real-time qPCR normalization during Al-induced programmed cell death in peanut. Biol. Plant. 63, 237-246 (2019).

35. Kotrade, P., Sehr, E. M., Wischnitzki, E. \& Brüggemann, W. Comparative transcriptomics-based selection of suitable reference genes for normalization of RT-qPCR experiments in drought-stressed leaves of three European Quercus species. Tree Genet. Genomes $15,1-12(2019)$.

36. De-Almeida, M. R. et al. Reference gene selection for quantitative reverse transcription-polymerase chain reaction normalization during in vitro adventitious rooting in Eucalyptus globulus Labill. BMC Mol. Biol. 11, 73 (2010).

37. Chen, H. et al. Reference genes selection for quantitative gene expression studies in Pinus massoniana L. Trees 30, 685-696 (2016).

38. Yang, Z. Y., Zhang, R. \& Zhou, Z. Identification and validation of appropriate reference genes for gene expression analysis in Schima superba. Genes 2, 732 (2021).

39. Delporte, M. et al. Selection and validation of reference genes for quantitative real-time PCR analysis of gene expression in Cichorium intybus. Front. Plant Sci. 6, 651 (2015).

40. Zhang, C. et al. Identification of suitable reference genes for gene expression normalization in the quantitative real-time PCR analysis of sweet Osmanthus (Osmanthus fragrans Lour.). PLoS ONE 10, e0136355 (2015).

41. Shu, S. et al. Effects of exogenous spermidine on photosynthetic capacity and expression of Calvin cycle genes in salt-stressed cucumber seedlings. J. Plant. Res. 127, 763-773 (2014).

42. Pettengill, E. A., Parmentier-Line, C. \& Coleman, G. D. Evaluation of qPCR reference genes in two genotypes of Populus for use in photoperiod and low-temperature studies. BMC. Res. Notes 5, 1-12 (2012).

43. Sperry, J. S. Evolution of water transport and xylem structure. Int. J. Plant Sci. 164, S115-S127 (2003).

44. Nakano, Y., Yamaguchi, M., Endo, H., Rejab, N. A. \& Ohtani, M. NAC-MYB-based transcriptional regulation of secondary cell wall biosynthesis in land plants. Front. Plant Sci. 6, 288 (2015).

45. Jiang, C., Gu, X. \& Peterson, T. Identification of conserved gene structures and carboxy-terminal motifs in the Myb gene family of Arabidopsis and Oryza sativa .L ssp. indica. Genome Biol. 5, R46 (2004).

46. Zhang, Y. et al. MYB56 encoding a R2R3 MYB transcription factor regulates seed size in Arabidopsis thaliana. J. Integr. Plant Biol. 55, 1166-1178 (2013).

47. Vilarrasa-Blasi, J. et al. Regulation of plant stem cell quiescence by a brassinosteroid signaling module. Dev. Cell. 30, 36-47 (2014).

48. Lee, D. K., Geisler, M. \& Springer, P. S. LATERAL ORGAN FUSION1 and LATERAL ORGAN FUSION2 function in lateral organ separation and axillary meristem formation in Arabidopsis. Development 136, 2423-2432 (2009).

49. Gomez, M. D. et al. Characterization of constricted fruit (ctf) mutant uncovers a role for AtMYB117/LOF1 in ovule and fruit development in Arabidopsis thaliana. PLoS ONE 16, e18760 (2011).

50. Alonso-Peral, M. M. et al. The microRNA159-regulated GAMYB-like genes inhibit growth and promote programmed cell death in Arabidopsis. Plant Physiol. 154, 757-771 (2010).

51. Xie, Z. et al. Role of the stomatal development regulators FLP/MYB88 in abiotic stress responses. Plant J. 64, 731-739 (2010).

52. Kim, J. H. et al. Loss of the R2R3 MYB, AtMyb73, causes hyper-induction of the SOS1 and SOS3 genes in response to high salinity in Arabidopsis. J. Plant Physiol. 170, 1461-1465 (2013).

53. Jung, C. et al. Overexpression of AtMYB44 enhances stomatal closure to confer abiotic stress tolerance in transgenic Arabidopsis. Plant Physiol. 146, 623-635 (2008).

54. Kirik, V. et al. Two novel MYB homologues with changed expression in late embryogenesis-defective Arabidopsis mutants. Plant Mol. Biol. 37, 819-827 (1998).

55. Mengiste, T. et al. The BOTRYTIS SUSCEPTIBLE1 gene encodes an R2R3MYB transcription factor protein that was required for biotic and abiotic stress responses in Arabidopsis. Plant Cell 15, 2551-2565 (2003). 
56. Galli, M. et al. Soluble interleukin-2 receptor decrease in the sera of HIV-infected patients treated with zidovudine. AIDS 5, 1231-1235 (1991).

57. Song, K. et al. Cellular changes of tracheids and ray parenchyma cells from cambium to heartwood in Cunninghamia lanceolata. J. Trop. For. Sci. 23, 478-487 (2011).

58. Chen, L. et al. Validation of reference genes for RT-qPCR studies of gene expression in banana fruit under different experimental conditions. Planta 234, 377-390 (2011).

59. Gao, Z. H. et al. Selection and validation of reference genes for studying stress-related agarwood formation of Aquilaria sinensis. Plant Cell. Rep. 31, 1759-1768 (2012).

\section{Author contributions}

K.P.L designed the methodology, conceived the study and wrote the manuscript. W.L and G.Y.T conducted the statistical analysis and revised the manuscript. K.Y.H provided project support. All authors read and approved the manuscript.

\section{Funding}

This study is supported by Innovation-driven development project of Guangxi Zhuang Autonomous Region, China (GK AA17204087-2), National Natural Science Foundation of China (32060352), National Natural Science Foundation of Guangxi Zhuang Autonomous Region, China (2018GXNSFAA294083), Department of Human Resources and Social Security of Guangxi Zhuang Autonomous Region, China (GuiCaiSheHan[2018]112).

\section{Competing interests}

The authors declare no competing interests.

\section{Additional information}

Supplementary Information The online version contains supplementary material available at https://doi.org/ 10.1038/s41598-021-04406-1.

Correspondence and requests for materials should be addressed to K.-Y.H.

Reprints and permissions information is available at www.nature.com/reprints.

Publisher's note Springer Nature remains neutral with regard to jurisdictional claims in published maps and institutional affiliations.

(c) (i) Open Access This article is licensed under a Creative Commons Attribution 4.0 International License, which permits use, sharing, adaptation, distribution and reproduction in any medium or format, as long as you give appropriate credit to the original author(s) and the source, provide a link to the Creative Commons licence, and indicate if changes were made. The images or other third party material in this article are included in the article's Creative Commons licence, unless indicated otherwise in a credit line to the material. If material is not included in the article's Creative Commons licence and your intended use is not permitted by statutory regulation or exceeds the permitted use, you will need to obtain permission directly from the copyright holder. To view a copy of this licence, visit http://creativecommons.org/licenses/by/4.0/.

(c) The Author(s) 2022 